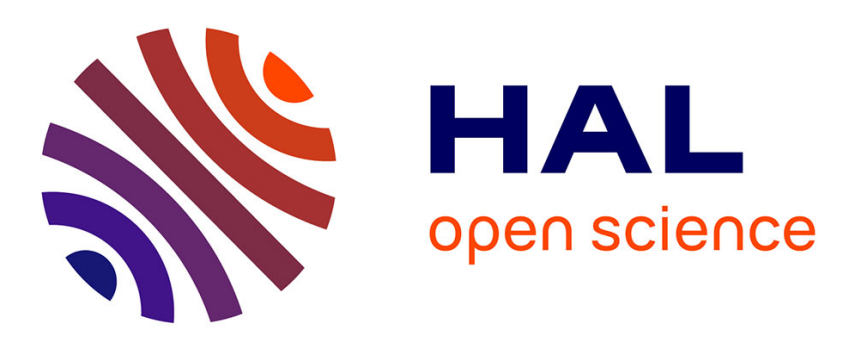

\title{
Effect of Remote Masking on Tactile Perception of Electrovibration
}

Milad Jamalzadeh, Cagatay Basdogan, Burak Güçlü

\section{To cite this version:}

Milad Jamalzadeh, Cagatay Basdogan, Burak Güçlü. Effect of Remote Masking on Tactile Perception of Electrovibration. IEEE Transactions on Haptics (ToH), In press, 10.1109/TOH.2020.3025772 . hal-03176841

\section{HAL Id: hal-03176841 \\ https://hal.science/hal-03176841}

Submitted on 22 Mar 2021

HAL is a multi-disciplinary open access archive for the deposit and dissemination of scientific research documents, whether they are published or not. The documents may come from teaching and research institutions in France or abroad, or from public or private research centers.
L'archive ouverte pluridisciplinaire HAL, est destinée au dépôt et à la diffusion de documents scientifiques de niveau recherche, publiés ou non, émanant des établissements d'enseignement et de recherche français ou étrangers, des laboratoires publics ou privés. 


\title{
Effect of Remote Masking on Tactile Perception of Electrovibration
}

\author{
Milad Jamalzadeh, Cagatay Basdogan, Senior Member, IEEE, and Burak Güçlü
}

\begin{abstract}
Masking has been used to study human perception of tactile stimuli, including those created by electrovibration on touch screens. Earlier studies have investigated the effect of in-site masking on tactile perception of electrovibration. In this study, we investigated whether it is possible to change the absolute detection threshold and intensity difference threshold of electrovibration at fingertip of index finger via remote masking, i.e. by applying a (mechanical) vibrotactile stimulus on the proximal phalanx of the same finger. The masking stimuli were generated by a voice coil (Haptuator). For 16 participants, we first measured the detection thresholds for electrovibration at the fingertip and for vibrotactile stimuli at the proximal phalanx. Then, the vibrations on the skin were measured at four different locations on the index finger of subjects to investigate how the mechanical masking stimulus propagated as the masking level was varied. Later, masked absolute thresholds of 8 participants were measured. Finally, for another group of 8 participants, intensity difference thresholds were measured in the presence/absence of vibrotactile masking stimuli. Our results show that vibrotactile masking stimuli generated sub-threshold vibrations around fingertip, and hence, probably did not mechanically interfere with the electrovibration stimulus. However, there was a clear psychophysical masking effect due to central neural processes. We measured the effect of masking stimuli, up to $40 \mathrm{~dB} \mathrm{SL}$, on difference threshold at four different intensity standards of electrovibration. We proposed two models based on hypothetical neural signals for prediction of masking effect on intensity difference threshold for electrovibration: amplitude and energy models. The energy model was able to predict the effect of masking more accurately, especially at high intensity masking levels.
\end{abstract}

Index Terms-Electrovibration, remote masking, Weber law. intensity difference threshold, absolute detection threshold, energy model, amplitude model.

\section{INTRODUCTION}

$\mathrm{T}$ ACTILE feedback is necessary to improve intuitiveness of human-machine interaction and/or as a substitution for other senses. In particular, displaying tactile feedback through touch screens is gaining importance due to its potential applications including those in consumer electronics, mobile computing, and the automotive industry. Implementing tactile feedback on touch screens requires a deeper understanding of how different tactile stimuli are perceived by humans, separately or simultaneously.

Tactile perception is mediated by psychophysical tactile channels, according to the four-channel theory of vibrotactile detection for glabrous skin [1]. Each tactile channel predominantly receives inputs from its corresponding receptor system and has its own frequency characteristics measured at threshold. In other words, human vibrotactile sensitivity varies as a function of stimulus frequency. This function has a U-shape at high frequencies with the lowest threshold at about $250 \mathrm{~Hz}$, but it is relatively flat at low frequencies. At suprathreshold levels, tactile perception can be quite complicated with the contributions of several psychophysical channels. The activations of individual channels have been studied mostly with the help of psychophysical masking effects. Tactile masking can be defined as the reduced ability to detect or discriminate a tactile pattern, when a second

- M. Jamalzadeh and C. Basdogan are with College of Engineering, Koc University, Istanbul, Turkey, 34450.

E-mail:mjamalzadeh17@ku.edu.tr, cbasdogan@ku.edu.tr

- B. Güçlü is with Institute of Biomedical Engineering, Boğaziçi University, Istanbul, Turkey, 34684.

Email: burak.guclu@boun.edu.tr

Manuscript received April 19, 2005; revised August 26, 2015. pattern is available in close temporal or spatial proximity to the first one [2]. The earlier psychophysical studies have investigated the effects of masking on absolute thresholds, difference thresholds, magnitude estimation, and localization of tactile patterns. Masking effects typically depend on the relative differences between the frequencies, intensities, spatiotemporal positions and durations of the target and the masking stimuli [3].

Different tactile masking types are usually defined based on their temporal order (see Vardar et al. [4]). As one of the most frequently applied techniques, forward masking has been shown to increase detection level for both low and high frequency stimuli, and can be used to selectively mask psychophysical channels [5], [6], [7], [8]. Forward masking can be explained by two alternative theories: persistence and neural adaption [9]. The persistence theory states that forward masking occurs because of persisting neural activity of masking stimulus after its offset. The neural adaption theory refers to changes in neural responses of target stimulus due to preceding stimulus. If the temporal order of the target and masking stimuli is reversed, one ends up with backward masking [10], [11]. Two mechanisms have been proposed for the backward masking effect: integration and interruption [12]. Integration theory assumes that the tactile stimuli presented in close temporal distance integrate into a composite representation, which obscures the perception of target stimuli [13]. Interruption theory proposes that the arrival of the second stimulus interrupts the processing of the target signal by diverting the processing resources away from the target [14]. In this study, we used pedestal masking technique in which masking stimuli are present 
before, during, and after the target stimuli. Therefore, almost all explanations, including those for forward and backward masking may be applicable here.

Masking effect highly depends on location and frequency of stimuli. The masking effect is maximized when both target and masking stimuli are activating the same channel [15], [16], and applied to the same location [17]. Verrillo et al. [17] studied the effects of locus and frequency of masking and target stimuli on thresholds. They observed shifts in threshold level for in-channel masking, when both target and masking stimuli were applied to a fingerpad. For the cross-channel case, some masking was observed when target stimulus frequency was in the range of the Pacinian channel and no effect when the target signal was stimulating non-Pacinian channels. For remote masking, if the masking stimulus was delivered to the thenar eminence and targeted to the fingerpad, the highest threshold shift was observed when both target and masking stimuli were exciting the Pacinian channel. Tanaka et al. [18] showed that a $50 \mathrm{~Hz}$ masking stimulus at forearm increased the threshold for detection of a $50 \mathrm{~Hz}$ stimulus at a fingertip, while no masking effect was observed when $200 \mathrm{~Hz}$ masking stimulus was used.

Tactile masking has great potential for the latest technological applications utilizing electrovibration. Electrovibration is one of several methods to display tactile feedback on a surface. This method has been recently integrated to touch screens [19], [20] where tactile effects are generated by modulating the friction between the user's fingertip and the touch screen. This can be achieved by applying a voltage signal to the conductive layer of a touch screen which is capacitively coupled with fingertip skin. The electrostatic force generated by this device causes a change in friction, and thus a tactile stimulus is felt during finger sliding. So far, only two research studies have been conducted to investigate tactile masking on touch screens actuated by electrovibration [4], [21]. One was on the effect of local masking on perception of electrovibration, with masking stimuli also presented as electrovibration [4]. The other study consisted of mechanical vibrations masking electrovibration detection also locally [21]. Vardar et al. [4] studied the effect of different masking methods, where the masking stimuli were presented as electrovibration, on electrovibration threshold of target stimuli. Their results showed that the highest effect is achieved in pedestal masking technique. They also showed that sharpness perception of virtual edges displayed on touch screens depends on the haptic contrast between background and foreground stimuli similar to the way it has been observed in vision studies. They demonstrated that this contrast varies as a function of masking amplitude and activation levels of frequencydependent psychophysical channels. On the other hand, Ryo et al. [21] attached piezoelectric actuators to a touch screen and investigated the effect of mechanical vibrations generated by these actuators on absolute and intensity difference thresholds of electrovibration.

In this study, we study the effect of directly applied mechanical vibrations on electrovibration absolute detection and intensity difference thresholds. However, unlike earlier studies [4], [21], our research focuses on remote masking. For this purpose, we applied mechanical vibrations on the proximal phalanx of index finger via a wearable voice coil as masking, while the finger was exploring an electrovibration stimulus on a touch screen. Considering that the popularity of wearable cutaneous devices, such as a haptic ring attached to the finger [22], [23], is increasing, the practical applications of remote vibrotactile masking could be in the areas of texture and shape display on touch screens via electrovibration.

We investigated the shift in absolute and intensity difference electrovibration thresholds as a function of masking intensity. In this regard, we first measured electrovibration and vibrotactile threshold levels of 16 subjects. Then, we investigated the masking effects on absolute detection and intensity difference thresholds relative to these thresholds. To rule out the possibility of a mechanical interaction at the fingertip, we also verified that the mechanical vibrations caused by the remotely applied masking stimuli were negligible close to the location where tactile stimulus was displayed by electrovibration.

To the best of our knowledge, this is the first study proposing a model based on Weber's law, which can explain the intensity difference threshold for electrovibration in the presence and absence of masking stimuli. There are two novelties in our model. First, unlike most of the previous studies, our model considers the background noise in Weber law and is able to calculate it indirectly. Second, while most of the existing studies use the amplitude of stimuli, or magnitude of some hypothetical neural activity for Weber's law, our results show that the signal energy (i.e. squared amplitude) of the hypothetical neural activity can predict the effect of masking on intensity difference threshold with higher accuracy compared to signal amplitude, especially at high intensity masking levels.

\section{Methods}

\subsection{Participants}

The experiments were conducted with two groups (G1, G2) of participants due to long duration of study. Both groups consisted of 5 male and 3 female participants. The average age of the participants in G1 and G2 were $29.3 \pm 6.2$ and 26.5 \pm 5.2 years, respectively. All participants were right handed except one in G1. All participants read and signed the consent form prior to the experiments. The experiments were approved by Ethical Committee for Human Participation of Koc University.

\subsection{Apparatus}

Figure 1 shows the setup used for the psychophysical experiments. It consisted of two actuators: a touchscreen (SCT3250, 3M Inc.) and a small high-bandwidth vibrotactile stimulator (Haptuator Mark II, Tactile Labs Inc.). The touchscreen was used to provide electrovibration stimuli on the fingerpad, while the vibrotactile stimulator was used to apply masking stimuli to the same finger. The input signals were generated by the analog output channels of a dataacquisition card (USB-6051, NI Inc.), and were amplified before driving the actuators. A voltage amplifier (E-314 D2, PI Inc.) and a custom-made power amplifier were used for the touchscreen and the vibrotactile stimulator, respectively. 


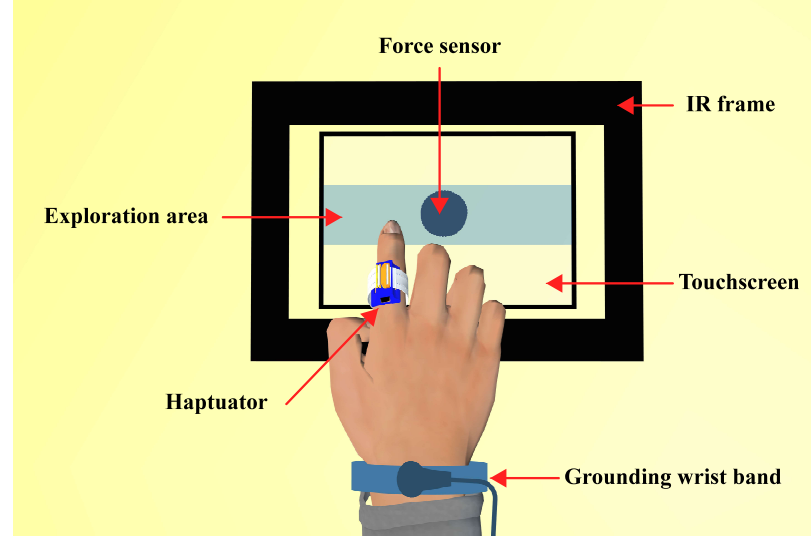

Fig. 1: An illustration of the setup used in the absolute detection and difference threshold experiments

The vibrotactile stimulator was placed inside a plastic case which was manufactured by 3D printing; it allowed direct contact with skin when mounted on the index finger. The case was fastened on the proximal phalanx of the dominant index finger by Velcro tapes, as shown in Figure 2. We followed a strict attachment protocol to maintain similar mechanical contact conditions. Using a grid paper on the plastic case, we achieved a specific amount of fastening for each participant in all experiments. A portable digital vibrometer, i.e. laser Doppler vibrometer (LDV), (PDV-100, Polytec Inc.) was used for measuring the mechanical vibrations generated by the vibrotactile stimulator and those propagated on the skin of the index finger. An IR-frame was placed over the touchscreen to detect finger position during movement. A force sensor (Nano17, ATI Inc.) was placed beneath the touchscreen to measure normal and tangential forces. A separate data acquisition card (PCI-6025E, NI Inc.) was used to record force data from the sensor. Throughout the experiments, participants wore a flexible wrist band for electrical grounding. They were also asked to put on noisecancelling headphones.

\subsection{Stimuli}

\subsubsection{Absolute Detection Threshold of Electrovibration and Vibrotactile Stimuli}

In absolute threshold experiment for both modalities (i.e. electrovibration and vibrotactile stimulation), each trial included two intervals: red and green. The test stimulus was randomly assigned to one of the intervals while the other one contained no stimulus. In the electrovibration threshold experiment, a $125 \mathrm{~Hz}$ sinusoidal voltage signal was displayed as the stimulus. This signal had a duration of $500 \mathrm{~ms}$ with additional $50 \mathrm{~ms}$ ramps at the beginning and at the end. In vibrotactile threshold experiment, the mechanical stimulator was excited by a $250 \mathrm{~Hz}$ sinusoidal signal with similar timing parameters as those used in the electrovibration threshold experiment. The reason for using half the frequency of mechanical vibrations in the electrovibration stimuli is due to the nonlinear relation between input voltage and output force (see Vardar et al. [20]). Vibrotactile thresholds are lowest at approximately 250 $\mathrm{Hz}$, due to the high sensitivity of the Pacinian channel.

\subsubsection{Vibration Propagation on Index Finger}

A series of sinusoidal signals at a frequency of $250 \mathrm{~Hz}$ were applied to vibrotactile stimulator to measure the propagated vibrations on the skin surface at four different locations of the index finger of participants (Figure 2). Duration of each signal was 2 seconds with a 2 second gap between them. The magnitude of the signals was set to $10 \mathrm{~dB}$ SL initially and then increased to $40 \mathrm{~dB}$ SL, where $\mathrm{dB}$ SL refers to decibels above the sensation, i.e. threshold, level. These intensity levels were adopted in the subsequent masking experiments.

\subsubsection{Absolute Detection Threshold of Electrovibration in the Presence of Vibrotactile Masking Stimuli}

In the absolute detection threshold experiment with masking, the electrovibration excitation signal waveforms were exactly the same as those used in the electrovibration threshold experiment. However, the vibrotactile masking stimuli with equal intensity were present in both intervals and for different experiments its intensities varied between 10, 20, 30 , and $40 \mathrm{~dB}$ SL in random order. The masking stimuli had sinusoidal waveforms with $50 \mathrm{~ms}$ on and off ramps and a total duration of 2 seconds including the ramps. The target signal was 0.5 seconds long and displayed in a time window overlapping the middle portion of the masking signal; both signals were centered within the interval.

\subsubsection{Electrovibration Difference Threshold Experiment}

In this experiment, the electrovibration stimuli were similar to the ones used in absolute threshold experiment, but they were presented in both intervals with different magnitudes. These experiments were conducted in the presence and absence of vibrotactile masking stimuli. For the difference threshold experiments with masking, the masking stimuli were the same as the ones used in absolute detection threshold experiment with masking. The vibrotactile masking stimuli with equal intensities were present in both intervals. The intensities were 20,30, and $40 \mathrm{~dB} \mathrm{SL}$, and they were presented to the participants in random order.

\subsection{Procedures}

\subsubsection{Absolute Detection Threshold of Electrovibration and Vibrotactile Stimuli}

We measured the absolute detection threshold levels separately for electrovibration and vibrotactile stimuli. In both experiments, the two-alternative-forced-choice method was used. Participants were asked to decide which interval contained the tactile stimulus. In the first trial, the amplitude of the signal was chosen well above the expected threshold level. Signal amplitudes for the following trials were set according to the modified three-down/one-up adaptive staircase method. Voltage applied to the actuator (touchscreen or vibrotactile stimulutor) was decreased after three correct answers, not necessarily given consecutively. Giving 1 incorrect answer resulted in an increase in voltage. The change in voltage (step size) was $5 \mathrm{~dB}$ until the second reversal. After the second reversal, the voltage change was in $1 \mathrm{~dB}$ steps. The experiment was terminated after 5 reversals in $2 \mathrm{~dB}$ range. The average of last 5 reversals was taken as the threshold value. In this way, the threshold value was 


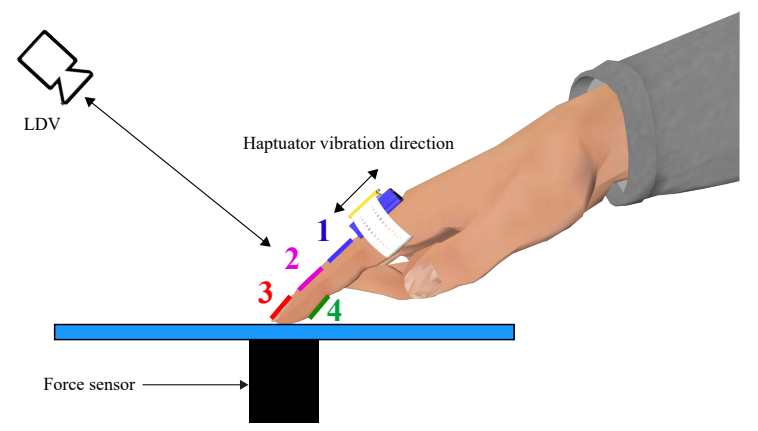

Fig. 2: Skin surface vibrations were measured at four different points on index finger using a digital vibrometer (LDV). At each point, a reflector was attached to reflect the emitted laser beam from the device perpendicularly. The measurements were conducted while the finger was stationary on the touch screen.

estimated at $75 \%$ correct probability of detection [24]. On average, each experiment lasted 15 minutes.

In the electrovibration detection threshold experiment, participants were asked to explore the touchscreen from left to right with a sliding speed of $50 \pm 12.5 \mathrm{~mm} / \mathrm{s}$, while their average normal force was kept in the range of 0.1-0.5 $\mathrm{N}$. In the vibrotactile detection threshold experiment, the finger was stationary on the touchscreen making a contact angle of 60 degrees, and the tactile stimuli were presented by the vibrotactile stimulator (this will be called Haptuator from now on). In this experiment, participants were also asked to keep the normal force applied to the touchscreen between 0.1 and $0.5 \mathrm{~N}$. First group of participants (G1) attended the absolute detection threshold experiments three times, on different days. For each participant, the average of three measurements was taken as the threshold value. We later used these values in the absolute detection threshold experiment in the presence of masking. Second group of participants (G2) attended the absolute detection threshold experiments only once. The vibrotactile and electrovibration threshold values from G2 were later used in the difference threshold experiment.

\subsubsection{Propagation of Mechanical Vibrations on Index Fin- ger}

Before conducting masking experiments, we investigated the vibration output of Haptuator on the index finger of participants. For each participant, the skin surface vibrations were measured at four points on the index finger as Haptuator excitation intensity was varied from 10 to 40 dB SL (Figure 2). For each intensity, the excitation signal was applied for 2 seconds with 2 second gaps between them. These measurements were important to verify that the propagation of the vibrations from the remote masking site was significantly attenuated at the electrovibration test site. Therefore, the remote masking could be mostly attributed to central neural processes, and not to a mechanical interference at the electrovibration site.
2.4.3 Absolute Detection Threshold of Electrovibration in the Presence of Vibrotactile Masking Stimuli

In this experiment, electrovibration threshold was measured at the fingertip, while mechanical vibrations were remotely applied by Haptuator for masking. The protocol of this experiment was the same as the one used in the threshold experiment for electrovibration. The only difference was the presence of masking stimuli at both intervals in all trials. We conducted the masking experiment using the pedestal masking method. In each experimental session, the masking intensity was kept constant in all trials. Masking stimuli were applied at 10, 20, 30, and $40 \mathrm{~dB}$ SL intensities in different sessions. For each masking level, the electrovibration threshold was measured three times, on different days, and the average of three measurements was taken as the masked threshold level.

\subsubsection{Difference Threshold of Electrovibration Stimulus in Absence and Presence of Vibrotactile Masking Stimulus}

In this experiment, we used two-alternative-forced-choice method to measure just noticeable difference (JND) of electrovibration stimulus intensity. In each trial, an electrovibration stimulus with fixed intensity, called reference stimulus, was present in one interval while in the other interval an electrovibration stimulus with higher intensity displayed (test stimulus). Participants were asked to decide which interval contained the stronger electrovibration stimulus. In the first trial, the difference between amplitude of reference and test stimuli was chosen large enough for a clear differentiation. For the following trials, the difference was set according to modified three-down/one-up adaptive staircase method. The difference was decreased after three correct answers, not necessarily consecutively. Giving 1 incorrect answer resulted in an increase in difference. The step change in difference was $5 \mathrm{~dB}$ until the second reversal. After the second reversal, the step size was set to $1 \mathrm{~dB}$. The experiment was terminated after 5 reversals in $2 \mathrm{~dB}$ range. The average of last 5 reversals was taken as the JND for difference threshold experiment. In this way, the JND value was estimated at $75 \%$ correct probability of detection [24]. These experiments conducted in the presence and absence of vibrotactile masking stimuli. Each participant of G2 performed the experiment in the presence and absence of vibrotacitle stimuli seperately once. On average, each experiment took 15 minutes to complete for each participant.

\subsection{Models}

According to Signal Detection Theory [25], neural activation patterns generated by tactile stimuli are perceived against background neural noise. Although levels of neural activity cannot be directly measured by psychophysical methods, their effects can be indirectly quantified by appropriate models. In each trial of the absolute detection threshold experiment, the neural activity during the test interval includes the effects of the tactile stimulus plus the background noise, while the neural activity during the other interval only includes the background noise. In the difference threshold experiment, both intervals include the effects of tactile stimuli in the presence of the background noise. Although we studied the absolute detection threshold and 
difference threshold of electrovibration separately, in fact, both of them can be considered as discrimination such that participants were looking for the interval with the stronger neural activity. Results of such discrimination experiments can be modeled by using Weber's law. According to Weber's law, the just noticeable difference in intensity has a linear relation with reference intensity. If psychophysical measures and neural codes for intensity are correlated, i.e. called consistency by Johnson et al. [26], one can easily represent the psychophysical measures as hypothetical neural activations. Indeed, the earlier studies showed that sensation magnitude and neural activity due to changes in vibrotactile intensity can be estimated reliably [8], [27], [28]. Therefore, in the models below, the letter $I$ refers to the hypothetical neural activation at the given intensity (generated by a certain driving voltage).

In order to apply Weber's law to both absolute and difference threshold experiments, we need to consider the background noise level $\left(I_{B G}\right)$ which is unknown during the experiments. In the electrovibration absolute detection threshold experiment, the reference is the background noise level while in the electrovibration difference threshold experiment, the reference is equal to the total neural activity level caused by reference electrovibration voltage $\left(I_{r e f}\right)$ and background noise $I_{B G}$.

In this section, we model all experimental outputs of this study by assuming a single Weber fraction $K$ for just noticeable change in the neural activity level, for both difference and absolute detection threshold experiments, in the absence of masking stimuli [29]. In other words, $I_{0}$ (the neural activity level caused by electrovibration stimulus at threshold level) is also defined as a just noticeable change from $I_{B G}$ :

$$
K=\frac{I_{V}-I_{r e f}}{I_{B G}+I_{\text {ref }}} \equiv \frac{I_{0}}{I_{B G}}
$$

where $I_{V}$ is the total neural activity level resulting from reference voltage $\left(I_{r e f}\right)$ plus just noticeable difference from the denominator $\left(I_{B G}+I_{r e f}\right)$. The two equalities in Equation 1 can be summarized in the following form

$$
\frac{I_{V}}{I_{0}}-1=\frac{I_{r e f}}{I_{0}}(1+K)
$$

Since the normal electrostatic force applied to a finger by electrovibration (and its frictional effect during tangential movement) is proportional to the second power of applied voltage, we assume that the neural activity levels are also related to the second power of applied voltage:

$$
\frac{I_{V_{A}}}{I_{V_{B}}} \equiv \frac{V_{A}^{2}}{V_{B}^{2}}
$$

Equation 3 refers to Weber's law applied equivalently to voltages driving the touchscreen. The signal amplitude model given above is defined based on such an equivalence, that hypothetical neural activations are linearly related to normal electrostatic forces. Another model, i.e. signal energy model, is also tested in this study. In that model, ratios of hypothetical neural activations are again assumed to be equivalent to a function of the ratios of applied voltages, however this time, Weber's law was applied to the signal energy levels of hypothetical neural activations, instead of their amplitude levels. Since the energy of a signal is proportional to the second power of its amplitude, the energy of the hypothetical neural activations caused by electrostatic force will be proportional to second power of electrostatic force, or equivalently fourth power of applied voltage.

$$
\frac{I_{V_{A}}}{I_{V_{B}}} \equiv \frac{V_{A}{ }^{4}}{V_{B}{ }^{4}}
$$

It is important to note that a signal energy is not necessarily related to the physical energy stored or dissipated in the setup. It is a mathematical construct (as used in the field of signal processing) for the hypothetical neural activations. Therefore, the signal energy model is merely a mathematical abstraction from the signal amplitude model. If there is a constant Weber fraction $\left(K_{\text {Amplitude }}\right)$ derived from the amplitude of hypothetical neural activations, then there will be a different constant Weber fraction $\left(K_{\text {Energy }}\right)$ derived from the energy of hypothetical neural activations to satisfy Equation 2.

Using the Equations 3 and 4, Equation 2 can be written in the following forms for the two models:

$$
\begin{gathered}
\frac{V^{2}}{V_{0}^{2}}-1=\frac{V_{R}^{2}}{V_{0}^{2}}\left(1+K_{\text {Amplitude }}\right) \\
\frac{V^{4}}{V_{0}^{4}}-1=\frac{V_{R}^{4}}{V_{0}^{4}}\left(1+K_{\text {Energy }}\right)
\end{gathered}
$$

where $V_{R}$ and $V_{0}$ are the reference and threshold-level electrovibration voltage amplitudes, respectively. $V$ represents the comparison voltage (just noticeable difference in voltage plus reference voltage) estimated by the unmasked psychophysical intensity discrimination experiments presented above.

To model the masking experiments, it is assumed that the background neural activity level is increased [29] due to masking. Therefore, for absolute threshold masking experiment, the Weber fraction can be written as:

$$
K=\frac{I_{0 M}}{I_{B G}+I_{M}}
$$

where $I_{M}$ is the increase in the background neural activity level caused by the masking stimulus. This masking effect typically increases as a function of the masking stimulus level [4], [8], [30]. $I_{0 M}$ is the increase in neural activity level caused by absolute threshold level of electrovibration in the presence of masking stimuli, for a particular level of masking. It is assumed that a single Weber fraction applies to both unmasked and masked threshold experiments. Furthermore, similar to Equation 1, Weber fraction is assumed to be the same for absolute and difference thresholds:

$$
K=\frac{I_{V M}-I_{r e f}}{I_{B G}+I_{M}+I_{r e f}} \equiv \frac{I_{0 M}}{I_{B G}+I_{M}}
$$

Where $I_{V M}$ is the total neural activity level resulting from reference voltage $\left(I_{r e f}\right)$ plus masked just noticeable difference from the denominator $\left(I_{B G}+I_{M}+I_{r e f}\right)$. Combining Equations 1 and 8 yields:

$$
\frac{I_{V M}}{I_{0}}-\frac{I_{0 M}}{I_{0}}=\frac{I_{r e f}}{I_{0}}(1+K)
$$


Based on the modeling assumptions given above (see Equations 3 and 4), Equation 9 can be related to touchscreen driving voltages as:

$$
\begin{gathered}
\frac{V_{M}^{2}}{V_{0}^{2}}-\frac{V_{0 M}{ }^{2}}{V_{0}{ }^{2}}=\frac{V_{R}^{2}}{V_{0}{ }^{2}}\left(1+K_{\text {Amplitude }}\right) \\
\frac{V_{M}{ }^{4}}{V_{0}{ }^{4}}-\frac{V_{0 M}{ }^{4}}{V_{0}{ }^{4}}=\frac{V_{R}{ }^{4}}{V_{0}{ }^{4}}\left(1+K_{\text {Energy }}\right)
\end{gathered}
$$

In Equations 10 and 11, $V_{M}$ represents the comparison voltage (just noticeable difference in voltage plus reference voltage) estimated by the masked psychophysical intensity discrimination experiments presented above. $V_{0 M}$ is the voltage for masked threshold. Amplitude model implies that there is a linear relation between $\left(V_{M}^{2}-V_{0 M}^{2}\right)$ and $V_{R}^{2}$ with a slope of $\left(1+K_{\text {Amplitude }}\right)$. On the other hand, the energy model suggests that there is a linear relation between $\left(V_{M}^{4}-V_{0 M}^{4}\right)$ and $V_{R}^{4}$ with a slope of $\left(1+K_{\text {Energy }}\right)$.

\section{Results}

\subsection{Absolute Detection Thresholds of Electrovibration and Vibrotactile Stimuli}

Table 1 shows the threshold values of each participant for electrovibration and vibrotactile stimuli applied at the fingertip and at the proximal phalanx, respectively. One concern before the vibrotactile threshold experiment was if the Haptuator case and fastening band would alter the coupling with the skin between different sessions. A oneway repeated measures ANOVA was conducted to compare the effect of detaching and attaching the Haptuator on vibrotactile threshold level before and after each session. There was no significant effect of this factor on vibrotactile threshold level $(F(2,12)=0.47, p=0.64)$.

\subsection{Vibration Propagation on Index Finger}

Figure 3 plots the mean skin vibration amplitudes measured at four different locations on the index finger of participants. This measurement showed that the maximum vibration was close to the Haptuator, and the vibrations were highly attenuated near the fingerpad. Vibrations at finger nail were slightly higher than those at the tissue around it (points 2 and 4), because of the higher transmission of vibrations in stiffer structures (bones and nail). The minimum vibration was observed beneath the index finger, close to fingertip. For all participants at $40 \mathrm{~dB} \mathrm{SL}$, the vibration amplitude of point 4 barely reached to the average threshold level of the Pacinian channel, which is about $-20 \mathrm{~dB}$ re $1.0 \mu \mathrm{m}$ peak [31]. Therefore, the mechanical effect of remote masking at such a high masking intensity level can be neglected at the fingertip for the subsequent masking experiment.

\subsection{Absolute Detection Threshold of Electrovibration in the Presence of Vibrotactile Masking Stimuli}

Figure 4 shows the mean threshold shift in detection threshold of electrovibration as a function of remote masking intensity. The threshold shift was calculated in $\mathrm{dB}$ using $20 \times \log _{10} A$, where $\mathrm{A}$ is the ratio of masked absolute threshold value to the unmasked one (given in excitation voltage of electrovibration). A simple linear model was used
TABLE 1: Electrovibration (EV) and vibrotactile absolute detection thresholds of participants measured at fingertip and at proximal phalanx respectively. The thresholds are given in terms of the output voltage of the EV amplifier and the input voltage of the Haptuator amplifier. Note that threshold values were measured only once for the subjects 9-16.

\begin{tabular}{|c|c|c|}
\hline Participant & $\begin{array}{c}\text { EV excitation } \\
\text { amplitude (volt) }\end{array}$ & $\begin{array}{c}\text { Haptuator amplifier } \\
\text { input (volt) } \times \text { 100 }\end{array}$ \\
\hline S1 & $8.13 \pm 0.53$ & $0.69 \pm 0.11$ \\
\hline S2 & $8.03 \pm 2.19$ & $1.75 \pm 0.23$ \\
\hline S3 & $9.00 \pm 1.36$ & $0.73 \pm 0.16$ \\
\hline S4 & $7.21 \pm 1.54$ & $0.60 \pm 0.16$ \\
\hline S5 & $7.15 \pm 1.43$ & $0.88 \pm 0.13$ \\
\hline S6 & $7.25 \pm 0.62$ & $0.53 \pm 0.06$ \\
\hline S7 & $7.75 \pm 1.74$ & $0.36 \pm 0.21$ \\
\hline S8 & $10.00 \pm 1.66$ & $0.66 \pm 0.10$ \\
\hline S9 & 10.00 & 2.90 \\
\hline S10 & 11.06 & 2.95 \\
\hline S11 & 7.85 & 2.70 \\
\hline S12 & 13.32 & 2.65 \\
\hline S13 & 10.30 & 2.52 \\
\hline S14 & 8.19 & 2.05 \\
\hline S15 & 7.30 & 1.73 \\
\hline S16 & 10.80 & \\
\hline
\end{tabular}

to predict the shift in electrovibration threshold for a given vibrotactile masking intensity $(F(1,2)=15.37, p=0.059)$, with $R^{2}=0.88$. Hence, the shift in electrovibration threshold, in $\mathrm{dB}$, was modeled by $-0.92+0.19 \times V$, where $V$ was the vibrotactile masking intensity in $\mathrm{dB}$ SL. According to this model, the electrovibration threshold shifted up by $0.19 \mathrm{~dB}$ for each $\mathrm{dB}$ increase in vibrotactile masking level intensity.

\subsection{Difference Threshold of Electrovibration Stimulus in Absence and Presence of Vibrotactile Masking Stimu- lus}

Figure 5 shows the mean difference limen of electrovibration as a function of remote masking level. Difference limens are also shown for the unmasked condition. When presenting the results, the just-noticeable difference in amplitude $(\delta V)$ was converted to the just-noticeable difference in intensity (difference limen, DL) by using

$$
D L=20 \log _{10} \frac{V_{R}+\delta V}{V_{R}}
$$

where $V_{R}$ is the amplitude of voltage in reference stimulus and $V_{R}+\delta V$ is the amplitude of voltage in the interval 


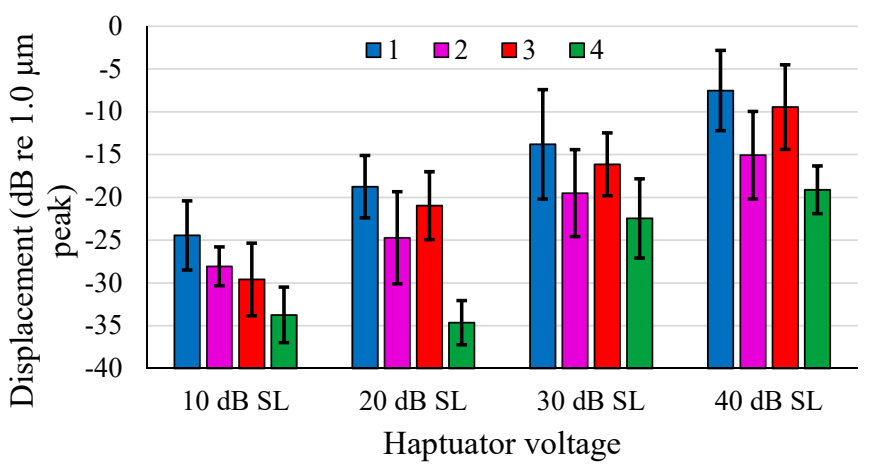

Fig. 3: Comparison of skin-surface vibration levels at different locations on the finger while Haptuator was actuated by a $250 \mathrm{~Hz}$ sinusoidal burst. Measurement was obtained at 10-40 dB SL Haptuator control voltage. Bar heights are the mean measurements across all participants and error bars are the standard deviations.

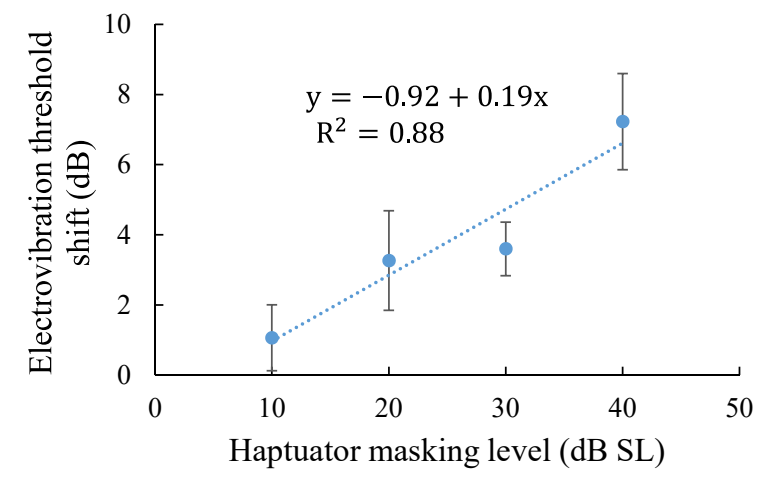

Fig. 4: Results of remote pedestal masking experiment. Mean electrovibration threshold shift $(\mathrm{dB})$ is shown as a function of remote vibrotactile masking intensity. The error bars depict the standard deviations.

with higher intensity (comparison stimulus). DL, as such, was calculated in $\mathrm{dB}$ units.

\subsection{Predictions by Amplitude and Energy Models}

Table 2 shows the results of fitting unmasked DL data to the models described in Equations 5 and 6, using simple linear regression method. The Weber fractions estimated by the signal amplitude and signal energy models are 0.30 and 0.87, respectively. These Weber fractions and the average shifted detection thresholds, which are shown in Figure 4, were further used to predict DLs of electrovibration in the presence of remote vibrotactile masking, since they were assumed not to change in the masked threshold and intensity discrimination experiments.

Model parameters estimated as explained above were used to generate the plots in Figure 5. Triangle symbols and asterisks in this figure show the DLs predicted by the signal amplitude and signal energy models, respectively. They were obtained by fitting data to Equations 10 and 11 . It is important to note that signal energy model predicts the psychophysical results better. In general, the signal amplitude model overestimated the DLs. There was especially
TABLE 2: Predictions of signal amplitude and energy models for JND experiments in the absence of masking stimuli. $K$ is the Weber fraction of the related model.

\begin{tabular}{|c|c|c|c|}
\hline Model & slope $=\mathbf{1}+\boldsymbol{K}$ & Weber fraction & $\boldsymbol{R}^{\mathbf{2}}$ \\
\hline Signal amplitude & 1.30 & 0.30 & 0.991 \\
\hline Signal energy & 1.87 & 0.87 & 0.993 \\
\hline
\end{tabular}

larger departure from experimental data as the masking level increased, e.g at $40 \mathrm{~dB}$ SL.

Figure 6 shows the psychophysical data in terms of the expressions given in Equations 10 and 11 for the signal amplitude model (Figure 6a) and the signal energy model (Figure 6b). Dashed blue straight lines are the predictions of the models based on the constant slopes reported in Table 2. In other words, as Figure 5 shows how the models perform psychophysically, Figure 6 shows how experimental data conform to constant slope assumption. As seen in Figure 6a, constant slope assumption is especially violated by the signal amplitude model at low reference levels for $20 \mathrm{~dB}$ SL masking level and at high reference levels for $40 \mathrm{~dB}$ SL masking level. On the other hand, the experimental data align well with the constant slope in the signal energy model.

\section{DISCUSSION}

\subsection{Mechanical vs. Neural Effects of Masking}

The results of vibration propagation on index finger showed that even at the highest magnitude of remote vibrotactile stimuli, those used for masking purpose, the amplitude of vibrations near the fingertip were negligible. However, due to technical difficulties with the digital vibrometer, we could not measure the vibrations exactly at the electrovibration site, i.e. the contact point between finger and touchscreen. Moreover, since the vibration source was on the dorsal side of the proximal phalanx, the mechanical waves probably had to move along the finger bones and skin to reach point 4 near the fingerpad (see Figure 3). Since the vibration amplitude at point 4 was barely reaching the average threshold of the Pacinian channel, we concluded that the vibration due to remote masking at the interface of fingerpad and touchscreen was at sub-threshold level. Jones and Sofia [32] investigated the propagation of travelling vibrotactile waves on the human skin at three sites (the palm of the hand, the forearm and the thigh) and found that the waves were attenuated by $8 \mathrm{~mm}$ on all the sites tested but was still measurable at $24 \mathrm{~mm}$. In our case, the distance between the Haptuator and point 4 was more than this distance (about $40 \mathrm{~mm}$ ). Hence, probably electrovibration and vibrotactile masking stimuli did not mechanically interfere, and the changes of absolute and difference threshold of electrovibration in the presence of masking stimulus are due to central neural processes within this study.

The results of our experiment on absolute detection threshold of electrovibration in the presence of vibrotactile masking stimuli showed that the absolute detection threshold of a $125 \mathrm{~Hz}$ electrovibration stimulus on the fingerpad of index finger increased almost linearly with increasing amplitude of a remote masking vibration at $250 \mathrm{~Hz}$ applied 


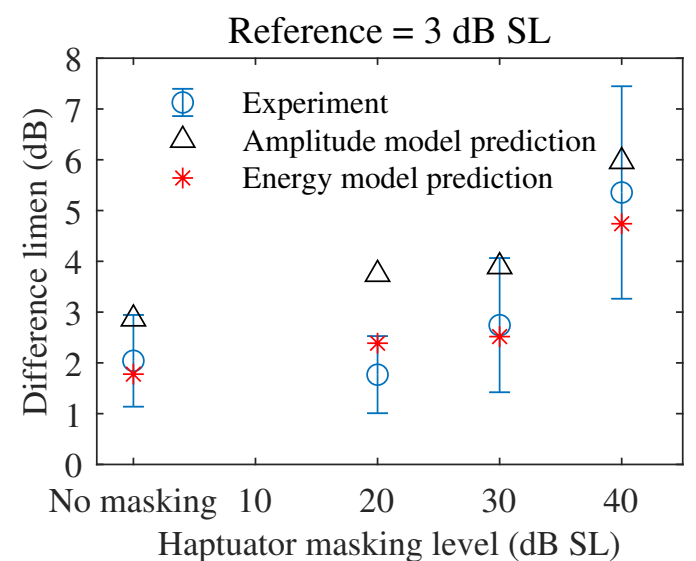

(a)

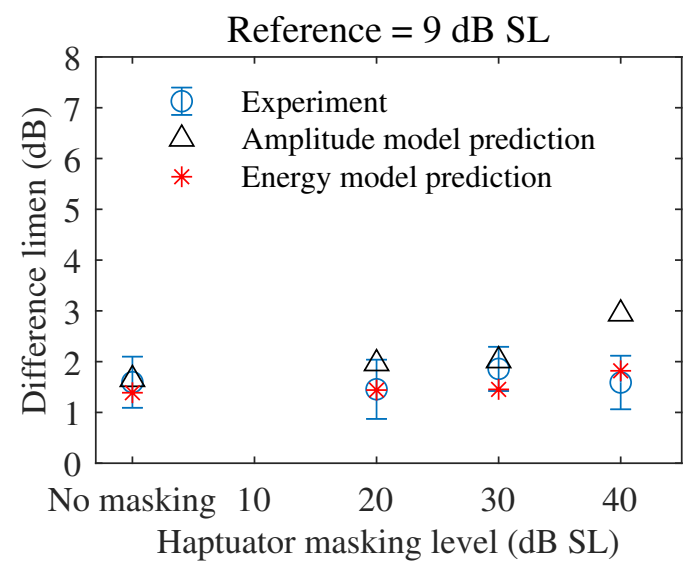

(c)

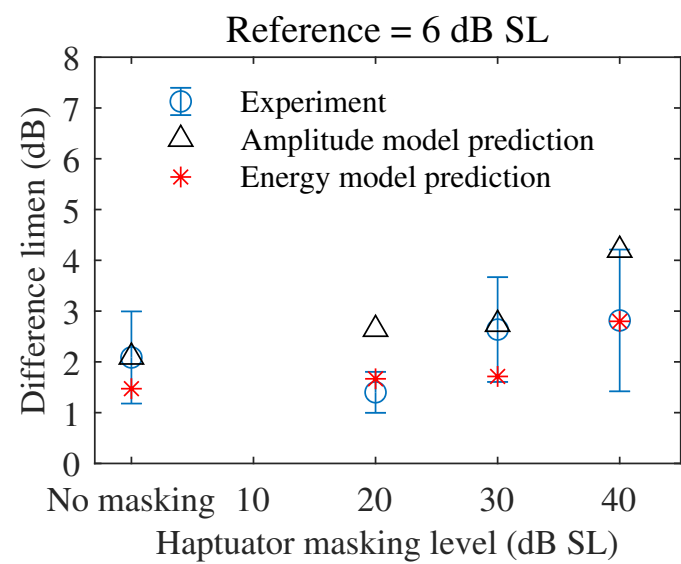

(b)

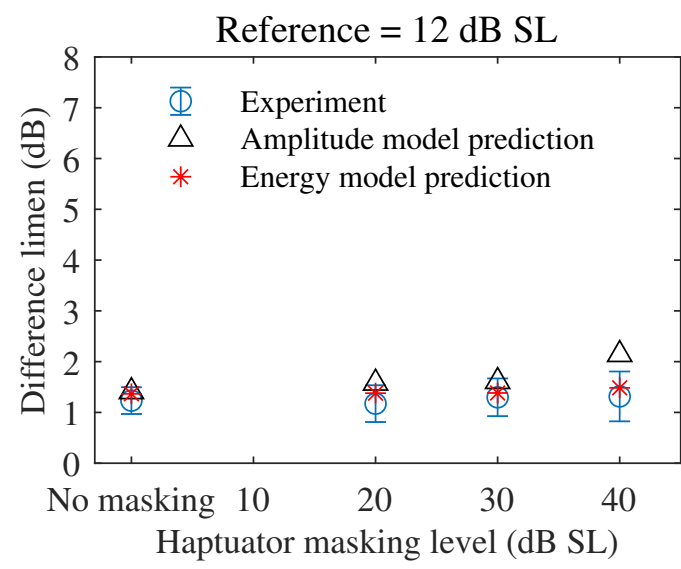

(d)

Fig. 5: Results of electrovibration intensity discrimination experiment and model predictions. At four different reference levels, mean difference limen (DL) is shown as a function of vibrotactile masking intensity. Leftmost data points are difference limens obtained without masking. The error bars depict the standard deviations. The asterisks and triangle symbols show the predictions of energy and amplitude models for the mean of difference limens respectively.

to proximal phalanx of the same finger. Table 3 compares the results of our experiment with the earlier studies in the same area. The threshold shift in our study is less than those obtained in earlier studies. We refer readers to our earlier publication [33] for the possible reasons of this difference.

\subsection{Modeling and Weber Fraction}

The results of difference threshold experiment in absence of masking stimuli are in line with earlier studies. Like earlier vibrotactile studies [7], [29], [34], the value of difference limen is relatively higher for lower reference levels (The average DLs were 2.04 and 2.09 for 3 and $6 \mathrm{~dB}$ SL reference levels, 1.6 and 1.23 for 9 and $12 \mathrm{~dB}$ SL ones, the difference is rather small; $\sim 1 \mathrm{~dB}$ ), which would result in higher Weber fractions. At first sight, it is tempting to attribute this deviation from Weber's law to not considering background noise during the calculation of the Weber fraction. As such, at high references, background noise would be negligible, but at low references, it may have an effect on Weber's law. However, Güçlü et al. [7] showed that the total spike activity from a mechanoreceptive afferent population, i.e. rapidly-adapting fibers, can also generate a similar devi- ation from Weber's law. Since this population does not have spontaneous activity, there is no 'neural noise'. It can be mathematically proven that many families of response measures defined over the population activity, e.g. concave up or power law, can generate such behavior in Weber fractions.

Nevertheless, a background noise is highly likely, albeit very difficult to measure, in the central nervous system. This is indeed the main tenet of Signal Detection Theory in psychophysics. An important contribution and novelty of the current work is the simplifying assumption that there is a unique, i.e. constant, Weber fraction applicable to absolute and difference thresholds, whether they are obtained with or without masking. By using the modeling approach presented here, all the thresholds could be predicted based on ratiometric measurements of touchscreen excitation voltages. Since the hypothetical neural activations were also put in ratiometric forms, the only additional assumption was the equivalence of the ratios between neural activations and electrostatic forces in the touchscreen governed by the excitation voltages. Two different relationships were used for this purpose: signal amplitude model and signal energy 


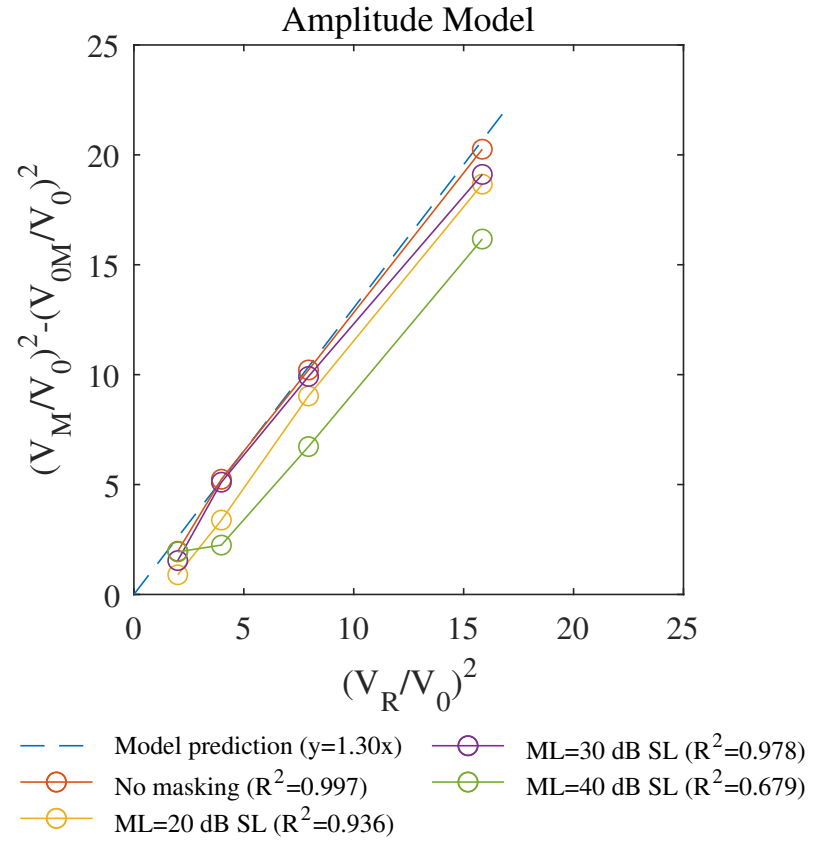

(a)

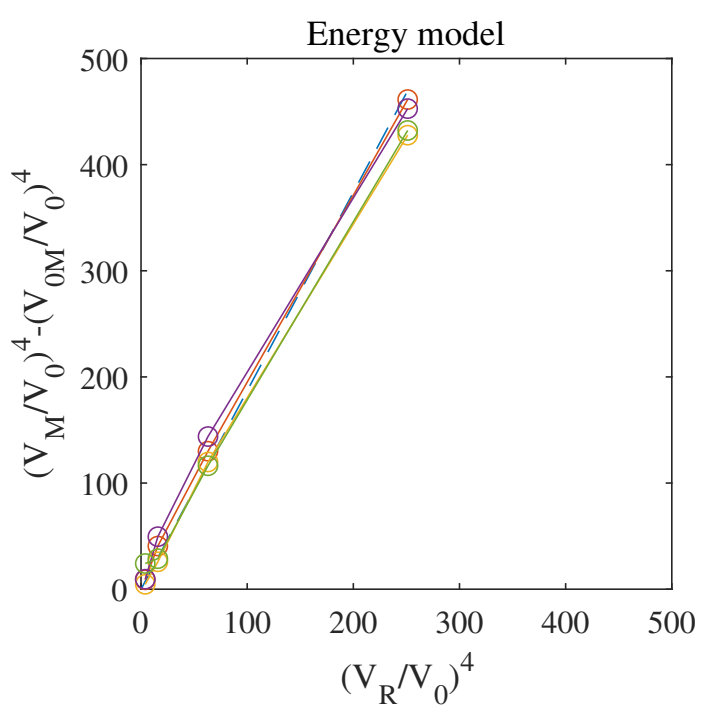

$\begin{array}{llll}- & \text { Model prediction }(\mathrm{y}=1.87 \mathrm{x}) & \bigcirc & \mathrm{ML}=30 \mathrm{~dB} \text { SL }\left(\mathrm{R}^{2}=0.989\right) \\ - & \text { No masking }\left(\mathrm{R}^{2}=0.997\right) & \bigcirc & \mathrm{ML}=40 \mathrm{~dB} \mathrm{SL}\left(\mathrm{R}^{2}=0.985\right) \\ - & \mathrm{ML}=20 \mathrm{~dB} \text { SL }\left(\mathrm{R}^{2}=0.984\right) & & \end{array}$ (b)

Fig. 6: Results of electrovibration intensity discrimination experiment for different vibrotactile masking levels (ML) are compared against constant slopes $(1+\mathrm{K})$ for the (a) signal amplitude model and (b) signal energy model.

TABLE 3: Comparison of electrovibration absolute threshold shifts (TS) between current and earlier studies

\begin{tabular}{|c|c|c|c|c|c|c|}
\hline \multirow{2}{*}{$\begin{array}{c}\text { Masking } \\
\text { location }\end{array}$} & Masking method & Masking signal & Target signal & Timing & $\begin{array}{c}\text { TS per dB } \\
\text { increase in } \\
\text { voltage }\end{array}$ & $\begin{array}{c}\text { TS per dB } \\
\text { increase in } \\
\text { force }\end{array}$ \\
\hline \multirow{2}{*}{ Local } & Electrovibration [4] & $\begin{array}{c}\text { Band limited noise } \\
\text { (75-150 Hz) }\end{array}$ & $125 \mathrm{~Hz}$ sinusoidal & Pedestal & 0.97 & 0.48 \\
\cline { 3 - 7 } & Mechanical [21] & $\begin{array}{c}120,180 \mathrm{~Hz} \\
\text { sinusoidal }\end{array}$ & $270 \mathrm{~Hz}$ sinusoidal & Simultaneous & 0.60 & 0.44 \\
\cline { 3 - 7 } & & $270 \mathrm{~Hz}$ sinusoidal & $270 \mathrm{~Hz}$ sinusoidal & Simultaneous & 0.60 & 0.66 \\
\hline \multirow{2}{*}{ Remote } & $\begin{array}{c}\text { Mechanical } \\
\text { (current study) }\end{array}$ & $250 \mathrm{~Hz}$ sinusoidal & $125 \mathrm{~Hz}$ sinusoidal & Pedestal & 0.19 & Unknown \\
\hline
\end{tabular}

model (Equations 3 and 4). With the help of these assumptions, we were able to find the unique Weber fraction to be about 0.30 in the signal amplitude model. This value is similar to the Weber fractions typically obtained in the earlier vibrotactile studies. For example, Craig found this value to be about 0.25 [29], [34] at $160 \mathrm{~Hz}$. Similarly, using the figures in Gescheider et al [35], Weber fraction can be calculated as 0.26 at $250 \mathrm{~Hz}$. Both of these studies and the current study targeted the Pacinian psychophysical channel, but the current work induced tactile stimuli by electrovibration in contrast. It is important to note that the Weber fraction may vary in different channels, or if more than one channel is activated. For example, for the Non-Pacinian I channel, the Weber fraction was reported to be in the range of $0.18-0.38$ at $40 \mathrm{~Hz}$ [7].

Although the signal energy model fits the data better, it yielded a Weber fraction of 0.87 which is somewhat inconsistent with the previous vibrotactile psychophysical studies. Although one may expect different Weber fractions, because of the difference in the mathematical forms of Equations 5 and 6, it would be a circular argument to calculate $K_{\text {Amplitude }}$ from $K_{\text {Energy }}$ based on those equations. Once the equivalence is assumed with either Equation 3 or 4 , a single representation should be selected. The discrepancy between the Weber fraction from the vibrotactile literature and the signal energy model presented here may be due to the modality difference of electrovibration. During the finger movement on touchscreen, electrovibration causes a friction modulation which would result in combined loading on the finger and complex mechanical waveform at the mechanoreceptor level. On the other hand, the mechanical waveforms generated during vibrotactile stimulation in the earlier studies are relatively simpler and dominantly in normal direction, hence afferent responses can be modeled rather precisely [28], [36]. Another reason for discrepancy may be the assumption of a unique Weber fraction. In 
Craig's study [29], a modeling approach based on amplitude was used for simultaneous remote masking with vibrotactile stimuli. A constant proportion of the masking effect was used in the equations to show that similar Weber fraction can be obtained for various masking levels and with no masking. However, this approach only worked when background noise contribution was included in the absolute threshold data, but not in the difference threshold data.

Nevertheless, the signal energy model has an additional conceptual benefit when considering the addition of hypothetical neural activations. We avoided using these terms (neural activations caused by background noise and masking stimuli) separately and always reduced them to ratiometric forms (Equations 2 and 9) for fitting, because they cannot be measured directly. This is a powerful approach since it isolates the psychophysical theory from the physically measurable quantities, i.e. voltages, by using equivalence functions (Equations 3 and 4). However, if one considers adding hypothetical neural activities due to background noise, target stimuli, or masking stimuli; a linear summation would be valid for very specific conditions only. For example, when the signals are of different waveforms, summation of signal amplitudes would not be meaningful. However, signal energies may be summed to represent the combined effect of multiple hypothetical neural activations.

\section{CONCLUSION}

To the best of our knowledge, our study is the first in the literature that propose a model to explain the differences in absolute detection and difference thresholds of electrovibration in the presence and absence of masking vibrotactile stimuli. We extended Craig's [29] model by constraining the equations to include a single Weber fraction for both absolute and difference thresholds experiments and by including the effect of background noise in the hypothetical neural activations. The link between hypothetical neural activations and touchscreen excitation voltages was established based on two alternatives. Signal amplitude model assumes that neural activation is linearly related to electrostatic force, and thus to squared voltage ratios. Signal energy model, on the other hand, assumes that this relationship is nonlinear, i.e. to the second power of electrostatic force, which yields ratios in the fourth power of excitation voltages. Therefore, in the former model, the combined effect of multiple neural activations can be considered as linear summation, and in the signal energy model as a type of nonlinear summation.

It is remarkable how theories on vibrotactile psychophysics could be applied to electrovibration sensation. We previously argued that electrovibration detection at 125 $\mathrm{Hz}$ (electrostatic force at $250 \mathrm{~Hz}$ ) was achieved by the Pacinian channel [20]. The current study also attempted to recruit the Pacinian channel for intensity discrimination and found a Weber fraction (by the signal amplitude model) similar to those reported in the literature. Furthermore, the effect of remote vibrotactile masking on electrovibration could be predicted based on the same theory. In other words, in-channel masking affected psychophysical sensitivity and discrimination in the given channel. It may be interesting to test the experiments and the model presented herein at different frequencies with appropriate reference amplitude levels to find out the contributions of different psychophysical channels to intensity discrimination by electrovibration.

Although remote masking leads to a less masking effect, it is a more appropriate way for studying the neural mechanism behind tactile perception of electrovibration. Remote masking is usually not susceptible to the complex problem of how target and masking stimuli interfere with each other mechanically in the skin to activate the mechanoreceptive afferents at the target site. In vibrotactile psychophysics literature, this is usually accomplished by using forward masking instead of simultananeous or pedestal masking (e.g. see [6]). However, the pilot experiments by Vardar et al. [4] showed that threshold shifts were less with forward masking by electrovibration at the same site (unpublished results); therefore, we used pedestal masking to obtain a robust effect. On the other hand, forward masking can still be effective if it is applied vibrotactually at the same site just before electrovibration as demonstrated by Ryu et al. [21], who indeed used simultaneous vibrotactile masking of electrovibration at the same site. The effect of forward masking is yet to be tested more systematically.

It is still not known how skin and peripheral afferents respond to complex tactile stimuli applied in both normal and tangential directions. From an application point of view, remote masking may still be more desirable because it can be applied simultaneously with the target signals in a more controlled and simple way. This would entirely decouple the mechanical and neural effects of masking. In this study, we used these advantages of remote masking to model how target and masking stimuli are processed within a sensory system without being worried about the mechanical aspects of the problem. Here, we just investigated tactile stimuli at the effective frequency of $250 \mathrm{~Hz}$ with a fixed duration. Further studies need to be conducted to investigate the effects of different stimulus frequencies and durations, and to verify whether standard psychophysical theories still apply.

\section{ACKNOWLEDGMENTS}

We acknowledge the technical support provided by Amirreza Aghakhani from Vibration and Acoustic Labratory of Koc University related to the digital vibrometer measurements.

\section{References}

[1] S. J. Bolanowski Jr, G. A. Gescheider, R. T. Verrillo, and C. M. Checkosky, "Four channels mediate the mechanical aspects of touch," The Journal of the Acoustical Society of America, vol. 84, no. 5, pp. 1680-1694, 1988.

[2] J. C. Craig, "Interference in localizing tactile stimuli," Perception $\mathcal{E}$ Psychophysics, vol. 45, no. 4, pp. 343-355, Jul 1989.

[3] G. Gescheider, K. Santoro, J. C. Makous, and S. Bolanowski, "Vibrotactile forward masking: effects of the amplitude and duration of the masking stimulus," The Journal of the Acoustical Society of America, vol. 98, no. 6, pp. 3188-3194, 1995.

[4] Y. Vardar, B. Güçlü, and C. Basdogan, "Tactile masking by electrovibration," IEEE Transactions on Haptics, vol. 11, no. 4, pp. 623-635, Oct 2018.

[5] M. Z. Yıldız, İ. Toker, F. B. Özkan, and B. Güçlü, “Effects of passive and active movement on vibrotactile detection thresholds of the pacinian channel and forward masking," Somatosensory $\mathcal{E}$ Motor Research, vol. 32, no. 4, pp. 262-272, 2015. 
[6] B. Güçlü and Ç. Öztek, "Tactile sensitivity of children: effects of frequency, masking, and the non-pacinian I psychophysical channel," Journal of Experimental Child Psychology, vol. 98, no. 2, pp. 113-130, 2007.

[7] B. Güçlü, "Deviation from weber's law in the non-pacinian I tactile channel: a psychophysical and simulation study of intensity discrimination," Neural Computation, vol. 19, no. 10, pp. 2638-2664, 2007.

[8] B. Güçlü and Ş. M. Dinçer, "Neural coding in the non-pacinian I tactile channel: A psychophysical and simulation study of magnitude estimation," Somatosensory \& Motor Research, vol. 30, no. 1, pp. 1-15, 2013.

[9] J. J. DiGiovanni, E. E. Lynch, N. K. Nagaraj, and D. T. Ries, "Dominance of persistence over adaptation in forward masking," Attention, Perception, \& Psychophysics, vol. 80, no. 7, pp. 1863-1869, 2018.

[10] M. Hollins, A. K. Goble, B. L. Whitsel, and M. Tommerdahl, "Time course and action spectrum of vibrotactile adaptation," Somatosensory \& Motor Research, vol. 7, no. 2, pp. 205-221, 1990.

[11] G. A. Gescheider and R. T. Verrillo, "Vibrotactile frequency characteristics as determined by adaptation and masking procedures," in Sensory Functions of the Skin of Humans. Springer, 1979, pp. 183-205.

[12] P. M. Evans and J. C. Craig, "Temporal integration and vibrotactile backward masking." Journal of Experimental Psychology: Human Perception and Performance, vol. 12, no. 2, pp. 160-168, 1986.

[13] D. W. Schultz and C. W. Eriksen, "Do noise masks terminate target processing?" Memory \& Cognition, vol. 5, no. 1, pp. 90-96, 1977.

[14] P. M. Evans, "Vibrotactile masking: Temporal integration, persistence, and strengths of representations," Perception $\mathcal{E}$ Psychophysics, vol. 42, no. 6, pp. 515-525, 1987.

[15] E. Bystrzycka, B. Nail, and M. Rowe, "Inhibition of cuneate neurones: its afferent source and influence on dynamically sensitive 'tactile' neurones," The Journal of Physiology, vol. 268, no. 1, pp. 251-270, 1977.

[16] D. Ferrington, B. Nail, and M. Rowe, "Human tactile detection thresholds: Modification by inputs from specific tactile receptor classes," The Journal of Physiology, vol. 272, no. 2, pp. 415-433, 1977.

[17] R. T. Verrillo, G. A. Gescheider, B. G. Calman, and C. L. Van Doren, "Vibrotactile masking: Effects of one and two-site stimulation," Perception \& Psychophysics, vol. 33, no. 4, pp. 379-387, 1983.

[18] Y. Tanaka, S. Matsuoka, W. M. B. Tiest, A. M. Kappers, K. Minamizawa, and A. Sano, "Frequency-specific masking effect by vibrotactile stimulation to the forearm," in International Conference on Human Haptic Sensing and Touch Enabled Computer Applications. Springer, 2016, pp. 156-164.

[19] O. Bau, I. Poupyrev, A. Israr, and C. Harrison, "Teslatouch: electrovibration for touch surfaces," in Proceedings of the 23nd annual ACM symposium on User Interface Software and Technology, 2010, pp. 283-292.

[20] Y. Vardar, B. Güçlü, and C. Basdogan, "Effect of waveform on tactile perception by electrovibration displayed on touch screens," IEEE Transactions on Haptics, vol. 10, no. 4, pp. 488-499, 2017.

[21] S. Ryu, D. Pyo, S.-C. Lim, and D.-S. Kwon, "Mechanical vibration influences the perception of electrovibration," Scientific Reports, vol. 8 , no. 1 , p. 4555,2018 .

[22] O. Ariza, P. Lubos, F. Steinicke, and G. Bruder, "Ring-shaped haptic device with vibrotactile feedback patterns to support natural spatial interaction," in Proceedings of the 25th International Conference on Artificial Reality and Telexistence and 20th Eurographics Symposium on Virtual Environments, ICAT-EGVE, vol. 15, 2015, pp. 175-181.

[23] C. Pacchierotti, G. Salvietti, I. Hussain, L. Meli, and D. Prattichizzo, "The hring: A wearable haptic device to avoid occlusions in hand tracking," in 2016 IEEE Haptics Symposium (HAPTICS). IEEE, 2016, pp. 134-139.

[24] J. J. Zwislocki and E. M. Relkin, “On a psychophysical transformed-rule up and down method converging on a $75 \%$ level of correct responses," Proceedings of the National Academy of Sciences, vol. 98, no. 8, pp. 4811-4814, 2001.

[25] G. A. Gescheider, Psychophysics: the Fundamentals. Psychology Press, 2013.

[26] K. O. Johnson, S. S. Hsiao, and T. Yoshioka, "Neural coding and the basic law of psychophysics," The Neuroscientist, vol. 8, no. 2, pp. 111-121, 2002.

[27] M. A. Muniak, S. Ray, S. S. Hsiao, J. F. Dammann, and S. J. Bensmaia, "The neural coding of stimulus intensity: linking the popula- tion response of mechanoreceptive afferents with psychophysical behavior," Journal of Neuroscience, vol. 27, no. 43, pp. 11687-11699, 2007.

[28] B. Güçlü and S. J. Bolanowski, “Modeling population responses of rapidly-adapting mechanoreceptive fibers," Journal of Computational Neuroscience, vol. 12, no. 3, pp. 201-218, 2002.

[29] J. C. Craig, "Vibrotactile difference thresholds for intensity and the effect of a masking stimulus," Perception \& Psychophysics, vol. 15, no. 1, pp. 123-127, 1974.

[30] B. Güçlü and S. J. Bolanowski, "Vibrotactile thresholds of the nonpacinian I channel: II. predicting the effects of contactor location on the phalanx," Somatosensory E Motor Research, vol. 22, no. 1-2, pp. 57-68, 2005.

[31] G. A. Gescheider, S. J. Bolanowski, and R. T. Verrillo, "Some characteristics of tactile channels," Behavioural Brain Research, vol. 148 , no. 1-2, pp. 35-40, 2004

[32] L. A. Jones and K. Sofia, "Measuring surface wave propagation during vibrotactile stimulation," in 2012 IEEE Haptics Symposium (HAPTICS). IEEE, 2012, pp. 457-461.

[33] M. Jamalzadeh, B. Güçlü, Y. Vardar, and C. Basdogan, "Effect of remote masking on detection of electrovibration," in 2019 IEEE World Haptics Conference (WHC). IEEE, 2019, pp. 229-234.

[34] J. C. Craig, "Difference threshold for intensity of tactile stimuli," Perception \& Psychophysics, vol. 11, no. 2, pp. 150-152, 1972.

[35] G. A. Gescheider, S. J. Bolanowski, R. T. Verrillo, D. J. Arpajian, and T. F. Ryan, "Vibrotactile intensity discrimination measured by three methods," The Journal of the Acoustical Society of America, 1990.

[36] B. Güçlü and S. J. Bolanowski, "Tristate markov model for the firing statistics of rapidly-adapting mechanoreceptive fibers," Journal of Computational Neuroscience, vol. 17, no. 2, pp. 107-126, 2004.

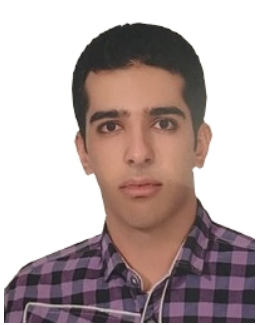

Milad Jamalzadeh received the BSc degree in mechanical engineering from Isfahan University of Technology, Isfahan, in 2017, the MSc degree in biomedical sciences and engineering from Koc University, Istanbul, in 2017. He is currently a PhD student in computational sciences and engineering program of Koc University. His research interests include haptic interfaces, tactile perception, and psychophysics.

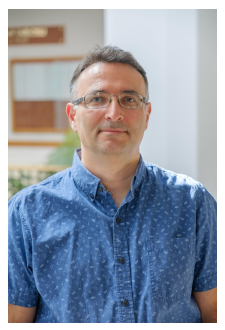

Cagatay Basdogan received the Ph.D. degree in mechanical engineering from Southern Methodist University in 1994. He is a faculty member in the mechanical engineering and computational sciences and engineering programs of Koc University, Istanbul, Turkey. He is also the director of the Robotics and Mechatronics Laboratory at Koc University. Before joining Koc University, he worked at NASAJPL/Caltech, MIT, and Northwestern University Research Park. His research interests include haptic interfaces, robotics, mechatronics, biomechanics, medical simulation, computer graphics, and multi-modal virtual environments. He is currently the associate editor in chief of IEEE Transactions on Haptics and serves in the editorial boards of IEEE Transactions on Mechatronics, Presence: Teleoperators and Virtual Environments, and Computer Animation and Virtual Worlds journals. He also chaired the IEEE World Haptics conference in 2011. 


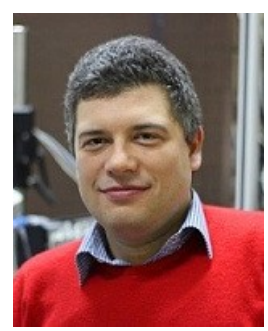

Burak Güçlü received a B.S. degree in Control and Computer Engineering from İstanbul Technical University in 1997 and an M.S. degree in Bioengineering from Syracuse University in 1999. He worked as a teaching assistant for Biocontrol Systems and Bioinstrumentation Laboratory. He recorded from tactile nerve fibers of cats at Institute for Sensory Research (ISR) in Syracuse, New York. In 1999-2000, Dr. Güçlü attended University of Michigan in Ann Arbor and studied sensory systems of insects at Advanced Technology Laboratories, and of guinea pigs at Kresge Hearing Research Institute using multi-electrodes. Then, he returned to ISR and worked on mathematical modeling of the sense of touch. He performed psychophysical experiments on human subjects and tested computational models. He received his Ph.D. degree in Neuroscience from Syracuse University in 2003. During his post-doctoral year, he studied speciesspecific vocalizations and recorded from the prefrontal cortex of awakebehaving macaque monkeys in the medical school of University of Rochester. Dr. Güçlü has been with Institute of Biomedical Engineering at Boügaziçi University since 2004. He is the director of Tactile Research Laboratory at the Institute and has founded the University Vivarium in Center for Life Sciences and Technologies Research. His research focuses on the transformation of tactile information from the periphery to the cortex, and it involves spike recordings from rat cortical neurons in the hindlimb area. He has recently completed a project to develop cortical neuroprostheses for tactile feedback, and is associated with an EU consortium on graphene electrodes for novel neural interfaces. 